\title{
Computed tomography-guided localization for multiple pulmonary nodules: a meta-analysis
}

\author{
Xia Tang ${ }^{1}$, Hong-Mei Jian ${ }^{1}$, Yi Guan², Jie Miao³ ${ }^{3}$ Xin Liang ${ }^{1}$ \\ ${ }^{1}$ Department of Oncology, Fengjie People's Hospital, Chongqing, China \\ 2Department of Endocrine, Fengjie People's Hospital, Chongqing, China \\ ${ }^{3}$ Department of Second Surgery, Fengjie People's Hospital, Chongqing, China
}

Videosurgery Miniinv 2021; 16 (4): 641-647

DOI: https://doi.org/10.5114/wiitm.2021.104199

\begin{abstract}
Introduction: Preoperative computed tomography (CT)-guided localization is commonly employed to facilitate video-assisted thoracoscopic surgery (VATS)-guided diagnostic wedge resection of pulmonary nodules (PNS).

Aim: The present meta-analysis was conducted with the goal of better evaluating the feasibility, safety, and clinical efficacy of preoperative CT-guided localization for multiple PNs (MPNS).

Material and methods: PubMed, Embase, and the Cochrane Library databases were reviewed in order to identify all relevant studies published as of June 2020. Random effects modeling was then used to evaluate the pooled data. The meta-analysis was conducted using Stata v12.0 software.

Results: Eight relevant studies were identified for inclusion in the present meta-analysis. In total, 345 patients with 799 PNs were treated with preoperative CT-guided localization and subsequent wedge resection. The overall pooled technical success rate based on the nodules and patients was calculated to be $97 \%$ (95\% confidence interval (CI): 0.94-0.99) and 92\% (95\% Cl: 0.86-0.97), respectively. The overall pooled incidence rate of complications was calculated to be $40 \%$ (95\% Cl: 0.16-0.63). Significant heterogeneity was observed among the studies for all the parameters described above $\left(I^{2}=74.5 \%, 77.7 \%\right.$, and $96.3 \%$, respectively). No significant differences were detected in the technical success of localization and incidence of complications between coil and hook-wire groups. The risk of publication bias in the reporting of technical success rate of localization based on both nodules and patients was found to be high ( $p<0.001$ and $<0.001$, respectively).

Conclusions: Preoperative CT-guided localization may be effective in guiding VATS-guided wedge resection in patients with MPNs.
\end{abstract}

Key words: computed tomography, lung nodule, multiple, localization.

\section{Introduction}

Preoperative computed tomography (CT)-guided localization is commonly employed to facilitate video-assisted thoracoscopic surgery (VATS)-guided diagnostic wedge resection of pulmonary nodules (PNs), achieving high rates of successful localization (89.6-100\%) and wedge resection (97-100\%), with an acceptable rate of complications (8.8-12.6\%) [1-3]. Localization methods usually involve methylene-blue, hook-wire, coils, and radio-labeling agents [1-7]. Relative to cases without localization, preoperative localization can significantly decrease the need for thoracotomy or VATS anatomical resection when diagnosing PNs [4].

Preoperative CT-guided localization of PNs has a number of advantages over other methods and is 
widely used for patients with multiple PNs (MPNs) [8-15]. Several groups have reported their experiences of one-stage localization for MPNs [12, 14, 15]. However, variability in the technical success of such localization has been reported, with studies employing different localization methods (methylene blue, hook-wire, or coils) reporting success rates of anywhere between $88 \%$ and $100 \%$ [8-15]. There is a similar degree of variability in the reported complication rates (13-90\%) [8-15]. As such, there is a clear need for other large-scale studies with large datasets to more accurately evaluate the feasibility and safety of preoperative CT-guided localization for MPNs.

\section{Aim}

The present meta-analysis was conducted with the goal of better evaluating the feasibility, safety, and clinical efficacy of preoperative CT-guided localization for MPNs.

\section{Material and methods}

\section{Study selection}

PubMed, Embase, and the Cochrane Library databases were searched for all relevant articles published up to June 2020, using the following search strategy: ((localization[Title/Abstract]) AND (nodule[Title/Abstract])) AND (multiple[Title/Abstract]).

Only studies that satisfied the following criteria were included in the review: (a) studies specifically focused on preoperative CT-guided localization (including staining materials, hook-wire, coil, and radio-label localization) for PNs; (b) patients with MPNs; (c) the diameter of each PN $\leq 30 \mathrm{~mm}$; and (d) studies should report the technical success of CT-guided localization.

Studies were excluded if they were: (a) case reports (only 1-3 cases); (b) conference abstracts; (c) nonhuman studies; or (d) reviews.

\section{Data extraction}

Two investigators independently extracted data from all studies; the corresponding author asked to resolve any discrepancies that arose. Baseline data, demographic information, and treatment-related data were extracted from all studies.

\section{Quality assessment}

All studies identified for inclusion in the present meta-analysis had a non-randomized design. Their quality was evaluated using the Newcastle-Ottawa scale [16]. Any study with a score $\geq 5$ was deemed to be of high quality [16].

\section{Endpoints}

The endpoints of this meta-analysis included the technical success of localization, technical success of wedge resection, and localization-related complications.

MPNs were defined as the number of PNs $>1$. Technical failure of localization was defined as the surgeon being unable to visualize the localized materials [9]. When the localization device was dislodged it was also considered a technical failure. Wedge resection was considered to be successful if the target nodule was identified in the wedge-shaped lung tissue [15].

\section{Meta-analysis}

Stata v12.0 software was used to conduct the present meta-analysis. Owing to presumed heterogeneity, a random-effects model was used to calculate the pooled results, with weighting applied according to the inverse variance of the included studies, where heterogeneity was assumed. Heterogeneity of the studies was evaluated using the $\mathrm{Q}$ test and measured using the $R^{2}$ statistic, with $R>50 \%$ indicative of substantial heterogeneity. Subgroup analysis was used to investigate sources of heterogeneity. $P$-value $<0.05$ was the threshold of significance. Egger's regression asymmetry test was used to evaluate the risk of publication bias.

\section{Results}

\section{Study characteristics}

In the initial literature search, 231 studies that were potentially relevant to the present analysis were identified. Of these, 8 were ultimately included in the final meta-analysis. Full details of the study selection process are presented in Figure 1.

The overall characteristics of the studies included in the present meta-analysis are shown in Table I. All studies included in the review were retrospective, with scores on the Newcastle-Ottawa scale in the range 6-8. In total, 345 patients with 799 PNs were treated with preoperative CT-guided localization and subsequent wedge resection. In four studies, coil localization was used [11, 12, 14, 15], 

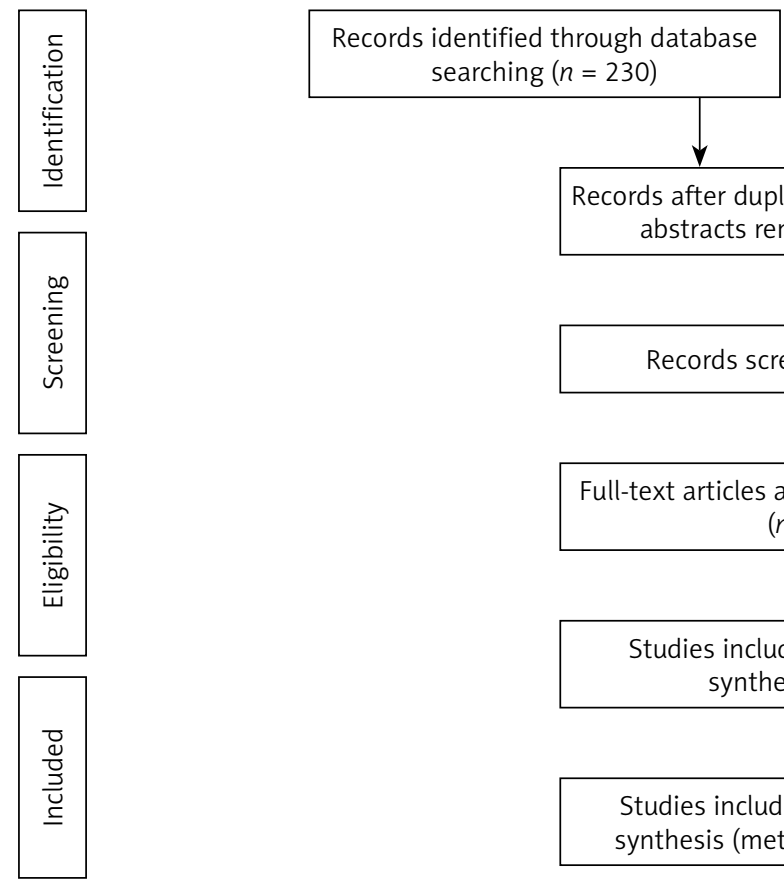
Additional records identified through other sources $(n=1)$

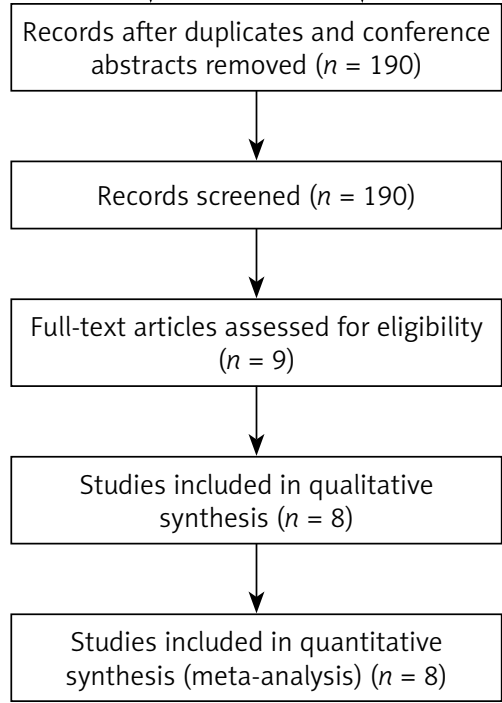

Figure 1. Flowchart of the meta-analysis

Table I. Characteristics of studies included in the meta-analysis

\begin{tabular}{|lcccccc|}
\hline Study & Year & Country & $\begin{array}{c}\text { Number of } \\
\text { patients }\end{array}$ & $\begin{array}{c}\text { Number of } \\
\text { nodules }\end{array}$ & Mean age [years] & $\begin{array}{c}\text { Newcastle- } \\
\text { Ottawa score }\end{array}$ \\
\hline Iguchi [8] & 2015 & Japan & 35 & 80 & 59.9 & 8 \\
\hline Tseng [9] & 2016 & Taiwan & 100 & 217 & 59.9 & 6 \\
\hline Kadeer [10] & 2018 & China & 76 & 194 & 55 & 8 \\
\hline Li [11] & 2018 & China & 51 & 126 & 58.9 & 8 \\
\hline Fu [12] & 2019 & China & 19 & 43 & 56.3 & 6 \\
\hline Sun [13] & 2019 & China & 16 & 34 & 55 & 8 \\
\hline Teng [14] & 2020 & China & 31 & 68 & Not given & 6 \\
\hline Yang [15] & 2020 & China & 17 & 37 & & 6 \\
\hline
\end{tabular}

hook-wire localization was used in $3[8,10,13]$, and one used methylene-blue localization [9]. The characteristics of the procedures are presented in Table II.

\section{Technical success of localization}

Data pertaining to the technical success of localization were reported in all studies, and calculated in relation to both nodules and patients. The overall pooled technical success rate based on nodules was calculated to be $97 \%$ (95\% confidence interval (Cl): 0.94-0.99, Figure 2 A). Significant heterogene- ity was detected among these studies $\left({ }^{2}=74.5 \%\right.$, $p=0.001)$. The overall pooled technical success rate based on patients was calculated to be $92 \%(95 \% \mathrm{Cl}$ : 0.86-0.97, Figure $2 \mathrm{~B}$ ), also with significant heterogeneity among the studies $\left(R^{2}=77.7 \%, p=0.000\right)$.

\section{Technical success of wedge resection}

The technical success of wedge resection was reported in 6 studies [9, 10, 12-15]. However, because 5 of those studies reported a technical success rate for wedge resection of $100 \%$ [9, 12-15], it was not possible to pool the results. 
Table II. Characteristics of procedures

\begin{tabular}{|c|c|c|c|c|c|c|c|}
\hline Study & $\begin{array}{l}\text { Mean lesion } \\
\text { size }\end{array}$ & $\begin{array}{l}\text { Localized } \\
\text { materials }\end{array}$ & $\begin{array}{c}\text { TS of } \\
\text { localization } \\
\text { (per nodule) }\end{array}$ & $\begin{array}{c}\text { TS of } \\
\text { localization } \\
\text { (per patient) }\end{array}$ & $\begin{array}{l}\text { TS of wedge } \\
\text { resection } \\
\text { (per nodule) }\end{array}$ & $\begin{array}{l}\text { TS of wedge } \\
\text { resection } \\
\text { (per patient) }\end{array}$ & $\begin{array}{c}\text { Complication } \\
\text { rate }\end{array}$ \\
\hline Iguchi [8] & $0.8 \mathrm{~cm}$ & Hook-wire & $96 \%$ & $91 \%$ & Not given & Not given & $90 \%$ \\
\hline Tseng [9] & $0.8 \mathrm{~cm}$ & $\begin{array}{c}\text { Methylene } \\
\text { blue }\end{array}$ & $99 \%$ & $98 \%$ & $100 \%$ & $100 \%$ & $55 \%$ \\
\hline Kadeer [10] & $0.7 \mathrm{~cm}$ & Hook-wire & $88 \%$ & $72 \%$ & $96 \%$ & $85 \%$ & $64 \%$ \\
\hline Li [11] & $0.8 \mathrm{~cm}$ & Coil & $96 \%$ & $90 \%$ & Not given & Not given & $24 \%$ \\
\hline $\mathrm{Fu}[12]$ & $0.7 \mathrm{~cm}$ & Coil & $98 \%$ & $95 \%$ & $100 \%$ & $100 \%$ & $16 \%$ \\
\hline Sun [13] & $0.7 \mathrm{~cm}$ & Hook-wire & $100 \%$ & $100 \%$ & $100 \%$ & $100 \%$ & $13 \%$ \\
\hline Teng [14] & $0.7 \mathrm{~cm}$ & Coil & $99 \%$ & $97 \%$ & $100 \%$ & $100 \%$ & $13 \%$ \\
\hline Yang [15] & $0.9 \mathrm{~cm}$ & Coil & $97 \%$ & $94 \%$ & $100 \%$ & $100 \%$ & Not given \\
\hline
\end{tabular}

TS-technical success.

\section{Complications}

The complication rate was reported in 7 studies [8-14]. The overall pooled complication rate was calculated to be $40 \%$ (95\% Cl: 0.16-0.63, Figure $2 \mathrm{C}$ ). Significant heterogeneity was detected among the studies $\left(I^{2}=96.3 \%, p<0.001\right)$.

\section{Subgroup analysis}

Subgroup analysis was then conducted for each endpoint variable for the different localization tech- niques (Table III). Only one study used methylene blue localization [9] and so it was excluded from the subgroup analysis. No significant differences were found in the technical success of localization and the incidence of complications between coil and hook-wire groups.

\section{Publication bias}

Publication bias analysis suggested that the risk of such bias relating to the reported complication

A

Study ID

ES (95\% Cl) Weight (\%)

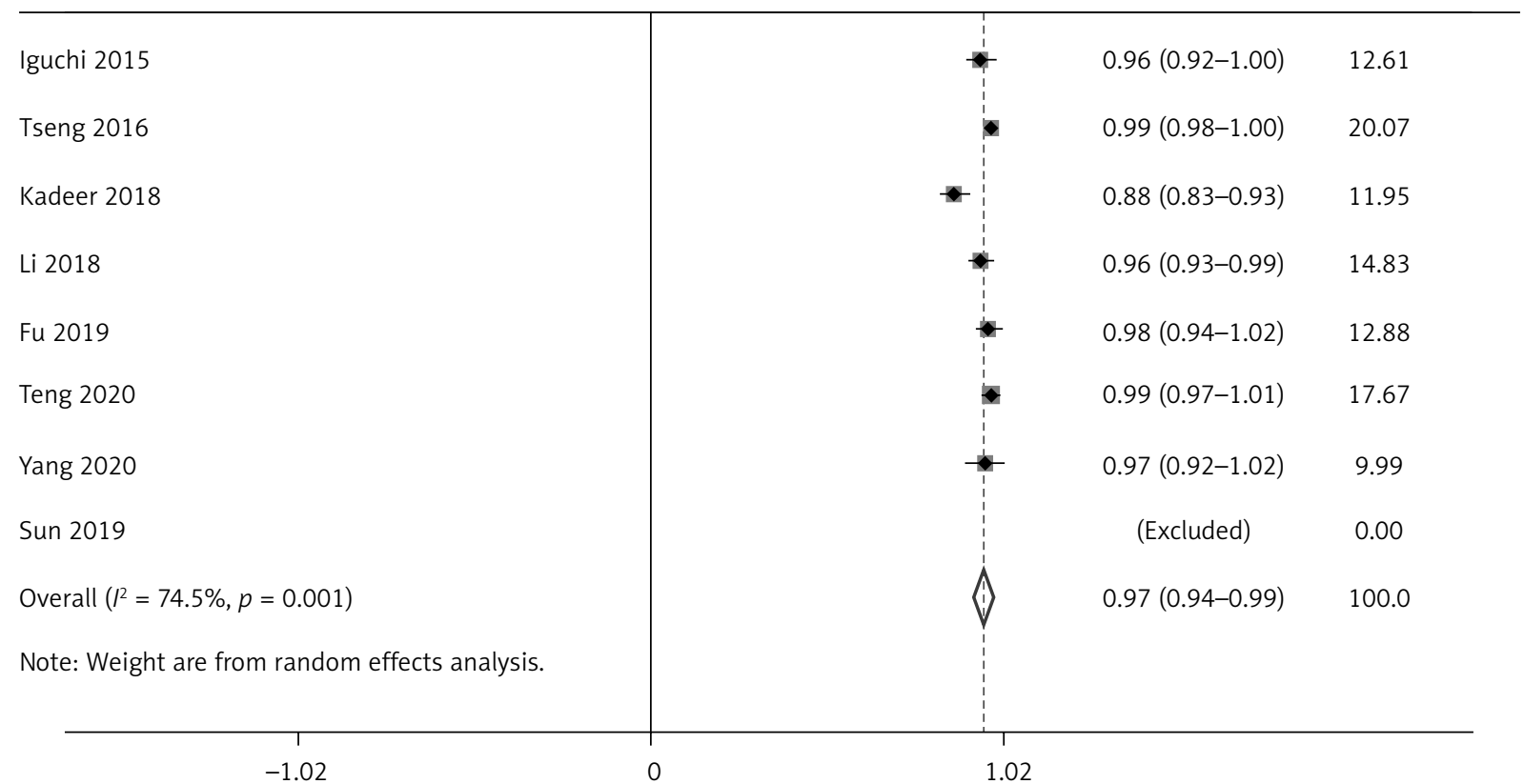

Figure 2. Forest plots of the technical success rate of localization (based on PNs) (A) 
B

Study ID

ES $(95 \% \mathrm{Cl}) \quad$ Weight $(\%)$

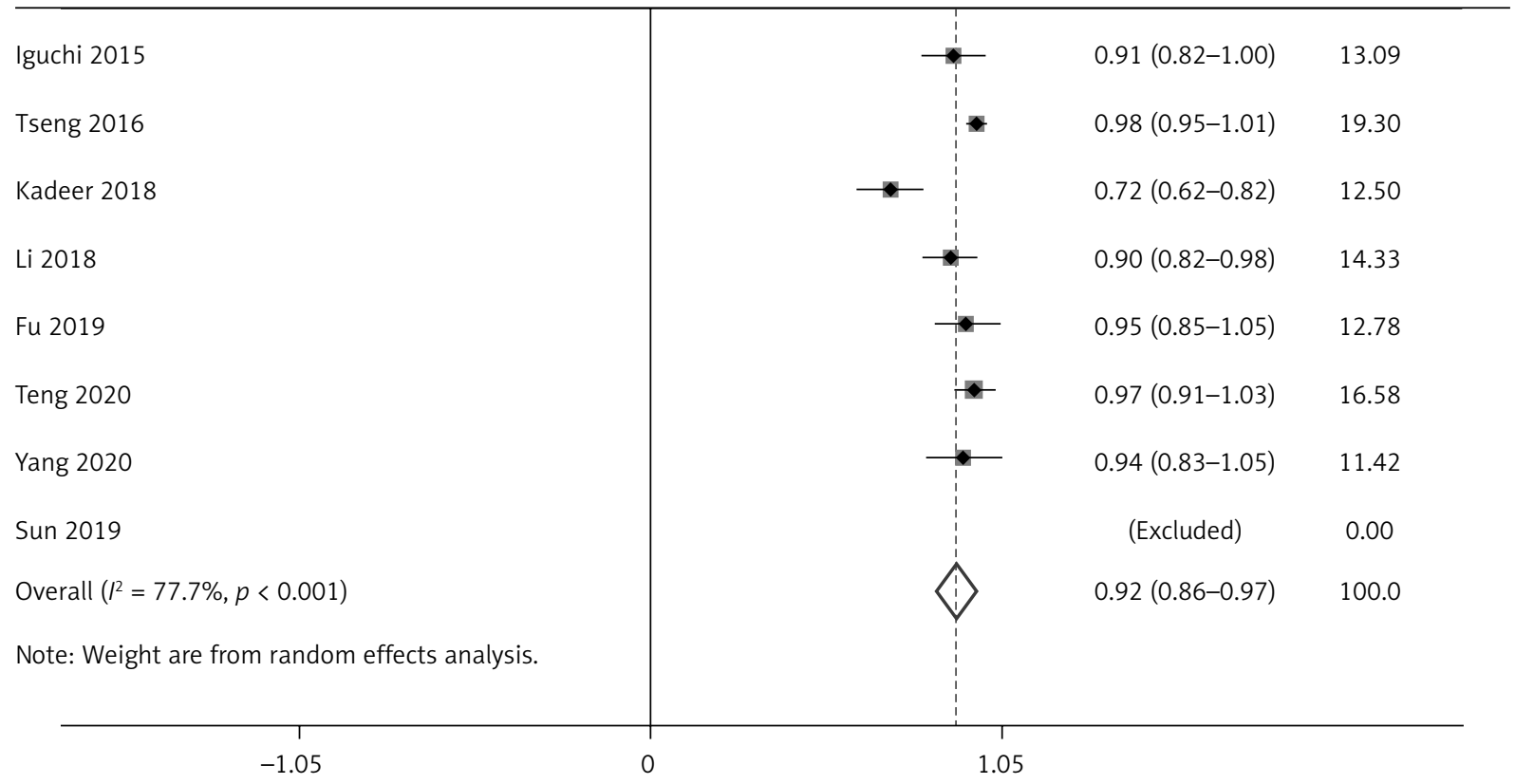

C

Study ID

ES $(95 \% \mathrm{Cl}) \quad$ Weight $(\%)$

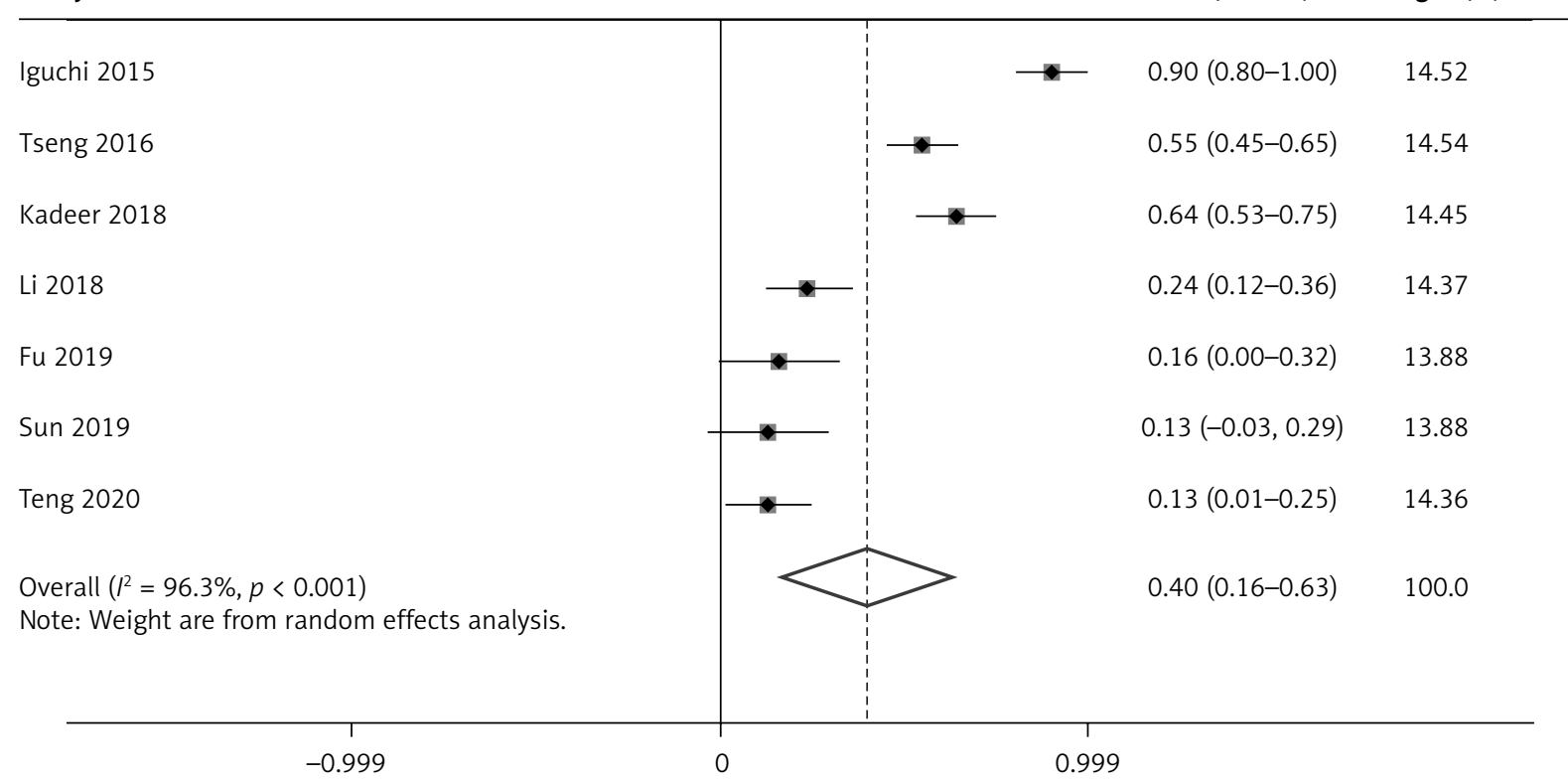

Figure 2. Cont. Technical success rate of localization (based on patients) (B) and complication rate (C)

rate was low $(p=0.351)$. In contrast, the risk of publication bias pertaining to the reported technical success rate of localization based on both nodules and patients was found to be high $(p<0.001$ and 0.001 , respectively).

\section{Discussion}

In the present meta-analysis, the feasibility, safety, and clinical efficacy of preoperative CT-guided localization for MPNs were evaluated. The overall results of our analysis were relatively positive, with a pooled 
Table III. Subgroup analyses

\begin{tabular}{|lccc|}
\hline Variable & $\begin{array}{c}\text { TS of } \\
\text { localization } \\
\text { (per nodule) }\end{array}$ & $\begin{array}{c}\text { TS of } \\
\text { localization } \\
\text { (per patient) }\end{array}$ & $\begin{array}{c}\text { Complication } \\
\text { rate }\end{array}$ \\
\hline Total & $97 \%$ & $92 \%$ & $40 \%$ \\
\hline $\begin{array}{l}\text { Localized } \\
\text { materials: }\end{array}$ & & & \\
\hline $\begin{array}{l}\text { Coil } \\
\text { Hook-wire }\end{array}$ & $98 \%$ & $95 \%$ & $18 \%$ \\
\hline$P$-value & 0.104 & $82 \%$ & $56 \%$ \\
\hline TS - technical success. & 0.117 & 0.163 \\
\hline
\end{tabular}

technical success rate of $97 \%$ and $92 \%$, respectively, for localization in PN- and patient-based analyses. These results may indicate that it is feasible to simultaneously localize MPNs in a single-stage procedure. However, significant heterogeneity was also detected and it might decrease the reliability of the conclusion. The source of heterogeneity may come from the following factors: (a) all included studies are retrospective in nature; and (b) the included studies used different localized materials. Subgroup analysis demonstrated that the technical success rate for localization was not significantly different for coil and hook-wire groups. These results possibly indicate that the preoperative technical success of localization was not influenced by localization method. Although the study of Tseng et al. [9] was not included in the subgroup analysis due to the use of methylene blue, the technical success rates of localization in PN- and patient-based analyses were $99 \%$ and $98 \%$, respectively. Nevertheless, further meta-analysis which focuses on one unique localized material is still required.

The reasons for technical failure using the different localization methods differ. The failure of methylene blue localization was due principally to its diffusive nature [2,9]. The failure of hook-wire localization was due to it becoming dislodged while coils were occasionally inserted into the lung parenchyma causing the loss of intra-operative localization function [1]. Recently, Zhang et al. [17] reported their clinical experience of computed tomography in combination with intraoperative noninvasive percutaneous ultrasonic localization of PNs with a high technical success rate of $95.6 \%$. Intraoperative ultrasound can provide real-time localization. However, this technique may not localize the MPNs simultaneously.

Although the pooled localization technical success rate was more than $90 \%$, the raw data of the studies included in the review indicated that the majority of studies had a $100 \%$ technical success for wedge resection [9, 12-15]. VATS-guided wedge resection was usually initially performed following preoperative localization [18]. If localization failed, wedge resection could be performed on tissue identified by palpation of the nodule or coil $[1,9,12,14$, 15]. Therefore, wedge resection was possible even if preoperative localization failed, although this resulted in wasted surgical time. Fu et al. [12] and Teng et al. [14] found that preoperative CT-guided multiple coil localization could be used to guide single-stage VATS diagnostic wedge resection in patients with MPNs.

Some researchers also performed simultaneous VATS for PNs and synchronous mediastinal/esophageal lesions $[1,19]$. Under this condition, if the PNs were metastatic lesions or presented with groundglass PNs, only wedge resection was enough $[1,18]$. Therefore, preoperative CT-guided localization should be performed for such patients to increase the technical success rate of wedge resection.

In the meta-analysis, the pooled complication rate was $40 \%$. This rate is higher than that reported in previous studies (24-29\%) regarding preoperative localization for a single PN $[11,20]$. It seems reasonable that localization-related complications occurred more frequently in patients with MPNs. However, the significant heterogeneity still requires further trials to confirm this conclusion. Subgroup analysis revealed that the complication rate in the coil group was lower than in the hook-wire group, although not significantly so ( $18 \%$ vs. $56 \%, p=0.163)$. This may be as a result of the limited number of studies. A previous study of coil vs. hook-wire localization for PNs indicated that the total complication rate was lower in the coil group (25\% vs. $54 \%, p=0.04$ ) [21]. Further meta-analysis which compares the clinical effectiveness between different localized materials is required. Although the pooled complication rate was high in this meta-analysis, the majority of studies reported that most localization-related complications did not influence the subsequent VATS-guided resection procedure $[1,9,12,14,15]$.

There are a number of limitations to the present meta-analysis. Firstly, all studies included in the analysis were retrospective. In addition, we detected significant heterogeneity in the pooled results. Some variability may be attributable to the many potential confounding variables or covariates not reported in the manuscripts, such as operator experience, the direc- 
tion of approach, or needle tract depth. Furthermore, the meta-analysis included a variety of different localization methods, thus potentially biasing the results of technical success and complication rate analysis.

\section{Conclusions}

The results of the present meta-analysis suggest that preoperative CT-guided localization may be used effectively to guide VATS wedge resection in patients with MPNs. The use of a coil may decrease localization-related complications. Further meta-analysis which focuses on one unique localized material is still required.

\section{Conflict of interest}

The authors declare no conflict of interest.

\section{References}

1. Fu YF, Zhang M, Wu WB, et al. Coil localization-guided video-assisted thoracoscopic surgery for lung nodules. J Laparoendosc Adv Surg Tech A 2018; 28: 292-7.

2. Gruber-Rouh T, Naguib NNN, Beeres M, et al. CT-guided hookwire localisation prior to video-assisted thoracoscopic surgery of pulmonary lesions. Clin Radiol 2017; 72: 898.e7-8.e11.

3. Zhang ZD, Wang HL, Liu XY, et al. Methylene blue versus coilbased computed tomography-guided localization of lung nodules. Thorac Cardiovasc Surg 2020; 68: 540-4.

4. Finley RJ, Mayo JR, Grant K, et al. Preoperative computed tomography-guided microcoil localization of small peripheral pulmonary nodules: a prospective randomized controlled trial. J Thorac Cardiovasc Surg 2015; 149: 26-31.

5. Iguchi T, Hiraki T, Gobara H, et al. Transfissural route used for preoperative localization of small pulmonary lesions with a short hook wire and suture system. Cardiovasc Intervent Radiol 2015; 38: 222-6.

6. Xia FF, Shi YB, Wang T, et al. Computed tomography-guided transfissural coil localization of lung nodules. Thorac Cardiovasc Surg 2020; 68: 545-8.

7. Lin MW, Tseng YH, Lee YF, et al. Computed tomography-guided patent blue vital dye localization of pulmonary nodules in uniportal thoracoscopy. J Thorac Cardiovasc Surg 2016; 152: 535-44.e2.

8. Iguchi T, Hiraki T, Gobara H, et al. Simultaneous multiple preoperative localizations of small pulmonary lesions using a short hook wire and suture system. Cardiovasc Intervent Radiol 2015; 38: 971-6.

9. Tseng YH, Lee YF, Hsieh MS, et al. Preoperative computed tomography-guided dye injection to localize multiple lung nodules for video-assisted thoracoscopic surgery. J Thorac Dis 2016; 8 (Suppl 9): S666-71.

10. Kadeer X, Wang L, Zhang L, et al. Modified hook-wire placement technique for localizing multiple pulmonary nodules. J Surg Oncol 2018; 118: 1188-93.
11. Li F, Chen Y, Bian J, et al. Preoperative computed tomography-guided microcoil localization for multiple small lung nodules before video-assisted thoracoscopic surgery. Zhongguo Fei Ai Za Zhi 2018; 21: 857-63.

12. Fu YF, Gao YG, Zhang M, et al. Computed tomography-guided simultaneous coil localization as a bridge to one-stage surgery for multiple lung nodules: a retrospective study. J Cardiothorac Surg 2019; 14: 43.

13. Sun W, Zhang L, Wang L, et al. Three-dimensionally printed template for percutaneous localization of multiple lung nodules. Ann Thorac Surg 2019; 108: 883-8.

14. Teng F, Wu AL, Yang S, et al. Preoperative computed tomography-guided coil localization for multiple lung nodules. Ther Adv Respir Dis 2020; 14: 1753466620909762.

15. Yang ZJ, Liang YH, Li M, et al. Preoperative computed tomography-guided coil localization of lung nodules. Minim Invasive Ther Allied Technol 2020; 29: 28-34.

16. Stang A. Critical evaluation of the Newcastle-Ottawa scale for the assessment of the quality of nonrandomized studies in metaanalyses. Eur J Epidemiol 2010; 25: 603-5.

17. Zhang $\mathrm{Q}$, Wang Z, Jiang Y, et al. The application value of computed tomography in combination with intraoperative noninvasive percutaneous ultrasonic localisation of subpleural pulmonary nodules/ground-glass opacity in uniportal video-assisted thoracoscopy. Videosurgery Miniinv 2020. doi:10.5114/ wiitm.2020.101238.

18. Zhong L, Hu W, Li S, et al. Clinical study of video-assisted thoracoscopic surgery wedge resection in early-stage lung cancer by tumor mapping with indocyanine green. Videosurgery Miniinv 2019; 14: 545-50.

19. Zeng L, Zhuang R, Tu Z. Simultaneous uniportal video-assisted thoracic surgery for pulmonary nodules and synchronous mediastinal lesions. Videosurgery Miniinv 2020. doi:10.5114/ wiitm.2020.101240.

20. Chen S, Zhou J, Zhang J, et al. Video-assisted thoracoscopic solitary pulmonary nodule resection after CT-guided hookwire localization: 43 cases report and literature review. Surg Endosc 2011; 25: 1723-9.

21. Rostambeigi N, Scanlon P, Flanagan S, et al. CT fluoroscopic-guided coil localization of lung nodules prior to video-assisted thoracoscopic surgical resection reduces complications compared to hook wire localization. J Vasc Interv Radiol 2019; 30: 453-9.

Received: 1.01.2021, accepted: 23.01.2021. 